\title{
Maintaining Hair Inductivity in Human Dermal Papilla Cells: A Review of Effective Methods
}

\author{
Ehsan Taghiabadi ${ }^{a}$ Mohammad Ali Nilforoushzadeh ${ }^{a}$ Nasser Aghdami ${ }^{b}$ \\ aSkin and Stem Cell Research Center, Tehran University of Medical Sciences, Tehran, Iran; bepartment of \\ Regenerative medicine, Cell Science Research Center, Royan Institute for Stem Cell Biology and Technology, ACECR, \\ Tehran, Iran
}

\section{Keywords}

Cell culture · Dermal papilla · Hair follicle · Spheroid culture . Exosome

\begin{abstract}
The dermal papilla comprises mesenchymal cells in hair follicles, which play the main role in regulating hair growth. Maintaining the potential hair inductivity of dermal papilla cells (DPCs) and dermal sheath cells during cell culture is the main factor in in vitro morphogenesis and regeneration of hair follicles. Using common methods for the cultivation of human dermal papilla reduces the maintenance requirements of the inductive capacity of the dermal papilla and the expression of specific dermal papilla biomarkers. Optimizing culture conditions is therefore crucial for DPCs. Moreover, exosomes appear to play a key role in regulating the hair follicle growth through a paracrine mechanism and provide a functional method for treating hair loss. The present review investigated the biology of DPCs, the molecular and cell signaling mechanisms contributing to hair follicle growth in humans, the properties of the dermal papilla, and the effective techniques in maintaining hair inductivity in DPC cultures in humans as well as hair follicle bioengineering.
\end{abstract}

(c) 2020 S. Karger AG, Basel

\section{Introduction}

Hair loss is one of the most common complaints for which both male and female patients seek treatment. In general, hair is a feature of mammalians that plays a great role in their beauty, social acceptance, and self-esteem. Over the last decade, patent-pending statistics have shown increasing costs. The common treatments for hair loss include conventional chemical approaches such as minoxidil [1, 2], finasteride [2], herbal extracts [3-5], platelet-rich plasma (PRP) [6-9], adipose-derived stem cells [10-12], keratinocyte-conditioned media [13], and hair transplantation [14]. None of these methods, however, have been able to bring satisfactory results. The current advances in the field of cell therapy, tissue engineering, and regenerative medicine have brought new hope for hair loss treatment. Different research groups have carried out projects to create the hair organoid structure in the laboratory. Although research findings suggest that using stem cells and mice dermal papilla cells (DPCs) helps create the hair structure, these studies have not been successful in humans. The main reasons for the failure of human studies include the loss of trichogenic ability of the hair DPCs, the insufficient number of hair in people with severe hair loss, and the lack of appropriate medical 
culture media. The dermal papilla consists of mesenchymal cells in the hair follicle, which plays the main role in the regulation of hair growth. Maintaining the potential hair inductivity of the DPCs and the dermal sheath cells during cell culture is the most important factor in in vitro hair follicle morphogenesis and regeneration. In addition, the procedure of hair follicle morphogenesis and regeneration includes Wnt, Shh, Notch, bone morphogenetic protein (BMP), and other cell signaling pathways interaction between epithelial cells and DPCs [15]. The $\beta$-catenin signaling of dermal papilla controls other signaling pathways, including fibroblast growth factor (FGF) 7 and FGF10, which regulate the growth of epithelial cells in hair follicles. The Wnt cell signaling is the primitive dermal induction pathway for developing hair placodes. As an inhibitor of the Wnt signaling, Dickkopf-1 (Dkk-1) lies in the interfollicular dermis [16].

Subsequent studies have demonstrated that rodent DPCs lose their inductive properties after extended primary cell culture [17]. After in vitro subculture of hair DPCs expression, a lot of specific genes of these cells, such as Akp2, Alx3, and Alx4, reduced rapidly, accompanied by a loss of hair follicle inductivity [18]. The most significant changes in the DPC transcriptome occurred after only a brief period of in vitro two-dimensional (2D) culture, accounting for the rapid loss of their capacity for hair follicle induction [19].

Insufficient and weak functionality of the cultured human DPCs and development of appropriate in vitro DPC culture conditions are main challenges that restricted application of commonly developed cell-based strategies in hair loss clinical applications. In order to solve these problems, scientists are studying the procedure to modify the methods of in vitro DPC culture, which really stimulate the growth environment of hair follicles in vivo and maintain the hair inductive properties of DPCs.

Evaluation of hair inductivity of three-dimensional (3D) spheroid cultures of human DPCs in the mice showed hair follicle formation but no new hair follicles were observed with 2D cultures [20]. Maintenance of hair inductivity of DPCs during in vitro subculture can be restituted if appropriate molecular substances are provided; for example, Wnt and BMP cell signaling have been demonstrated to maintain hair follicle inductivity in cultured rodent papilla cells $[21,22]$. Similar strategies such as soluble growth factors or keratinocyte-conditioned medium have been used to maintain the hair inductivity of human DPCs [13] However, using these effective mice methods into the human model has been a rate-limiting step with this approach.

Maintaining Hair Inductivity in Human Dermal Papilla Cells
In recent years, the therapeutic potential of different sources of exosomes, such as mesenchymal stem cells (MSCs) and appropriate molecular substances, has been evaluated in regenerative medicine. Among the different effective approaches for maintaining the in vitro and in vivo hair inductivity of the DPCs, exosome-based treatment may be the most efficient method. The advantages of using exosomes in hair experiments and clinical application include induction of endogenous mechanisms, simple processing, long-term storage, and reduced risk of immune reactions. The results of studies have shown that exosomes can induce cell proliferation, migration, and angiogenesis and promote tissue repair.

\section{The Structure of Hair Follicles}

Hair follicles are living mini-organs that comprise dermal (mesenchymal) and epidermal (epithelial) cells. As a dermal fibroblast cell derived from the mesoderm layer, the DPC plays the main role in developing human hair follicles. The primary aggregation of DPCs in the dermal layer occurs in humans at approximately 60 days of age. The differentiation of epidermal matrix cells into diverse sheath and hair fiber compositions occurs with the propagation of the epidermal plug above the dermal papilla [23]. Three different buds, namely, sweat glands, the follicle bulge, and the sebaceous glands, extend out of the hair plug. The "arrector pili" muscle attaches to the bulge stem cell area and connects hair follicles to the connective tissue. The differentiation of the upper layers of the dermal papilla into the inner root sheath (IRS) and the outer root sheath (ORS) of the hair induces epidermal plug and communicates with DPCs. Undifferentiated cells play a major role in follicle formation in the surrounding matrix of the dermal papilla. According to Figure 1, the development process of a hair follicle comprises a continuous pathway involving induction, initiation, elongation, and differentiation. The basic construction of hair follicles is completed within 160 days of embryonic skin development. The skin consists of 3 types of hair follicle including lanugo, vellus, and mature hair. The lanugo hairs grown in the fetus replaced by vellus hairs after 9 months. The mature hair follicle due to androgen hormones substituted with vellus hair follicle [24]. Hair follicles in humans consist of the ORS, the IRS, the hair shaft, the bulge region, and the dermal papilla [25]. 


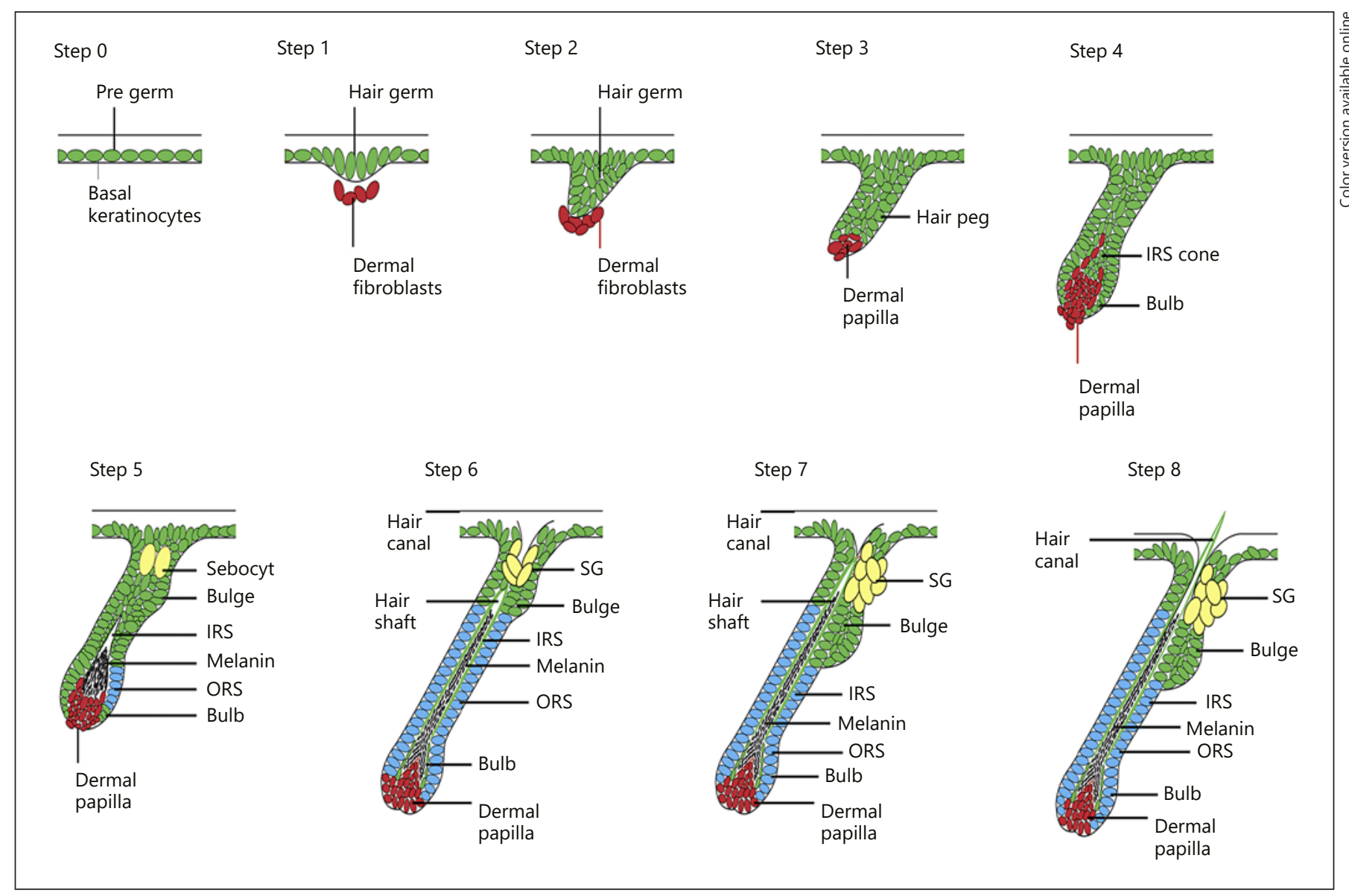

Fig. 1. The schematic steps of hair follicle morphogenesis. Step 0: no morphological indications of hair follicle formation; Step 1: placode induction; Step 2: condensation of the dermal fibroblast and elongation of the hair placode into the hair germ; Step 3: column arrangement of epidermal cells and hair peg formation; Step 4: development of the dermal condensate as the dermal papilla and covering it by the IRS; Step 5: development of hair follicles as the hair peg and formation of the first melanin; Step 6: formation of the hair shaft and enclosing the dermal papilla by epidermal cells; Step 7: the entry of the hair shaft into the hair canal; Step 8: finally, maximum elongation of hair follicles and emergence of hair through the epidermis. IRS, inner root sheath; SG, sebaceous gland.

\section{Hair Follicle Stem Cells and the Hair Cycle}

A major part of the slow-cycling ORS in the hair follicle with multipotent stem cells contributes to both the growth of hair follicles and the repair of a damaged epithelial layer. The morphogenesis of hair follicles and sebaceous glands is blocked in the absence of hair follicle stem cells (HFSCs). In the embryonic development stages, the hair follicle interaction between the skin epithelialmesenchymal section and the underlying dermal papilla plays a fundamental role in regulating the hair follicle cyclical activity [26]. Hair growth cycles of each mature follicle separate into 3 phases, that is, anagen (the growing phase), catagen (the regression phase), and telogen (the resting phase). In the catagen phase, hair stem cells are retained in the bulge region. The DPC signaling stimulates the activation of quiescent bulge stem cells in the catagen phase and increases the apoptotic rate of epithelial cells in the bulb and ORS regions. In the anagen phase, the interaction between bulge stem cells and hair germ stimulates the proliferation matrix of the new follicle to create a new hair filament [26, 27]. According to Figure 2 , hair follicles are inactive in the telogen phase of the hair cycle while a new hair starts to grow. Many paracrine factors and cell signaling molecules contribute to hair cycle stages in humans. 


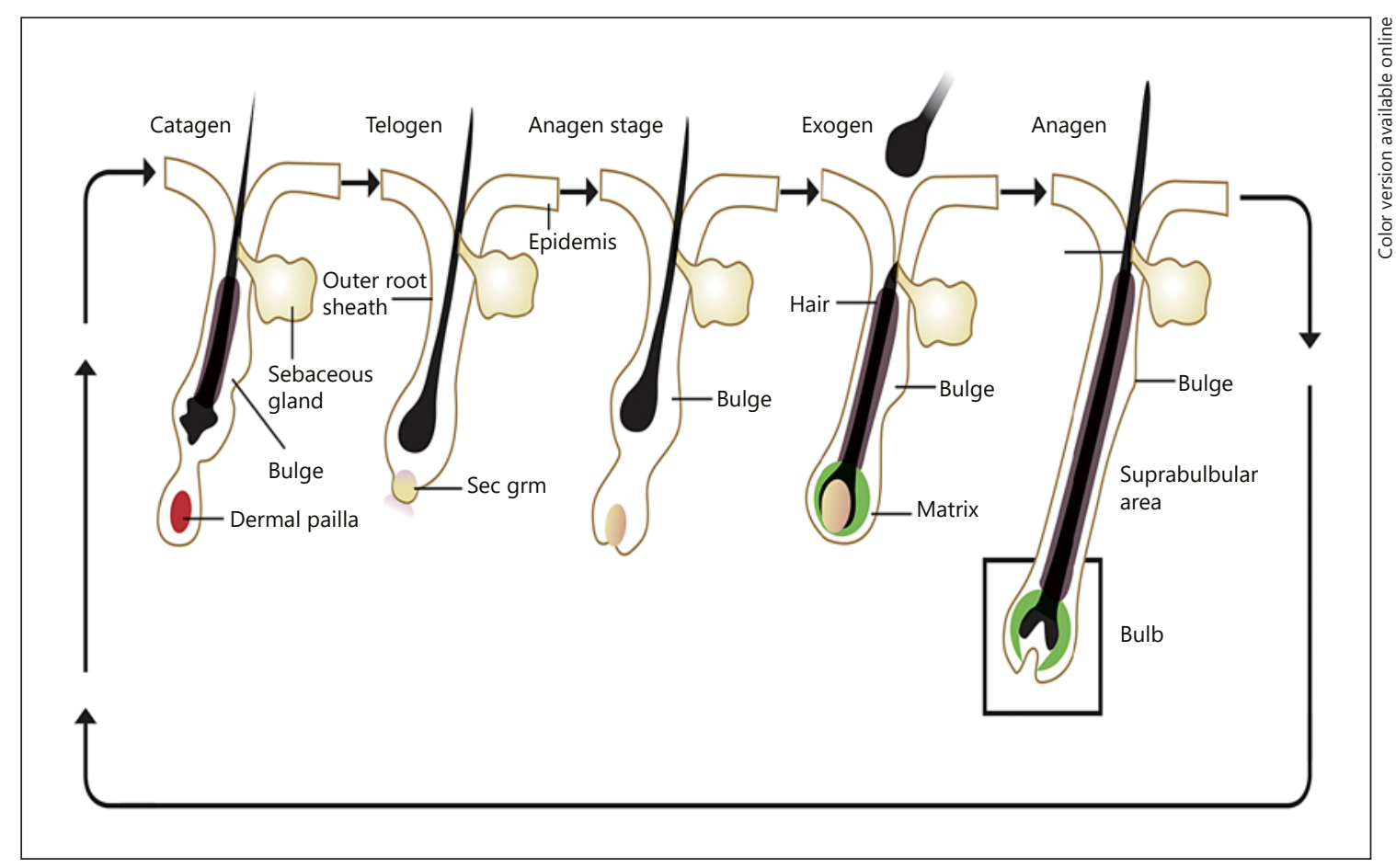

Fig. 2. The schematic of hair cycle stages. The intermittent cycles of the hair follicle growth include a growth phase (anagen), in which the interaction between bulge stem cells and DPCs creates a new hair filament; a regression phase (catagen), in which hair stem cells are retained in the bulge region and the apoptotic rate of epithelial cells increases; and a quiescent phase (telogen), in which hair follicles are completely inactive.

\section{The Signaling Pathway in the Morphogenesis and Development of Hair Follicles}

The development and morphogenesis of human hair follicles initiate with the main cell signaling pathways, including Wnt, sonic hedgehog (Shh), Notch, and the BMP. The Wnt pathway plays the role of a master regulator in the induction stage of the morphogenesis of hair follicles. At this stage, the Wnt cell signaling induces the overlying epithelial cells to develop the placode. At the organogenesis stage, the complex of epithelial cell signaling induces the proliferation of dermal cells, the formation of the dermal condensate, and the migration of dermal condensate into the dermal layer $[15,28]$. At the cytodifferentiation stage, covering the dermal condensate by follicular epithelial cells develops distinct dermal papilla. The Shh pathway plays the main role in the late-stage differentiation [29]. The prospects of HFSCs are determined by the Notch pathway. According to Figure 3, the BMP signaling regulates the cellular differentiation of hair follicles. Disorders of hair follicles are associated with the dysregulation of these signaling pathways [30].

Maintaining Hair Inductivity in Human Dermal Papilla Cells

\section{Growth Factors Involved in the Development of Hair Follicles}

A lot of growth factors and receptors regulate the development of hair follicles. Some of the growth factors contributing to the hair follicle growth and cell cycle regulation include epidermal growth factor (EGF), transforming growth factor- $\beta$ (TGF- $\beta$ ), insulin-like growth factor-1 (IGF-1), hepatocyte growth factor (HGF), keratinocyte growth factor (KGF), and vascular endothelial growth factor (VEGF). Any changes in the distribution of the relevant growth factor receptors and their expression levels can cause abnormalities in the growth and development of hair follicles [31, 32].

\section{Growth Factors}

\section{Epidermal Growth Factor}

As the main EGF receptor that regulates the hair follicle growth cycle, ErbB1 lies in the ORS of mature hair follicles. EGF binding to the ErbB1 receptor induces the 


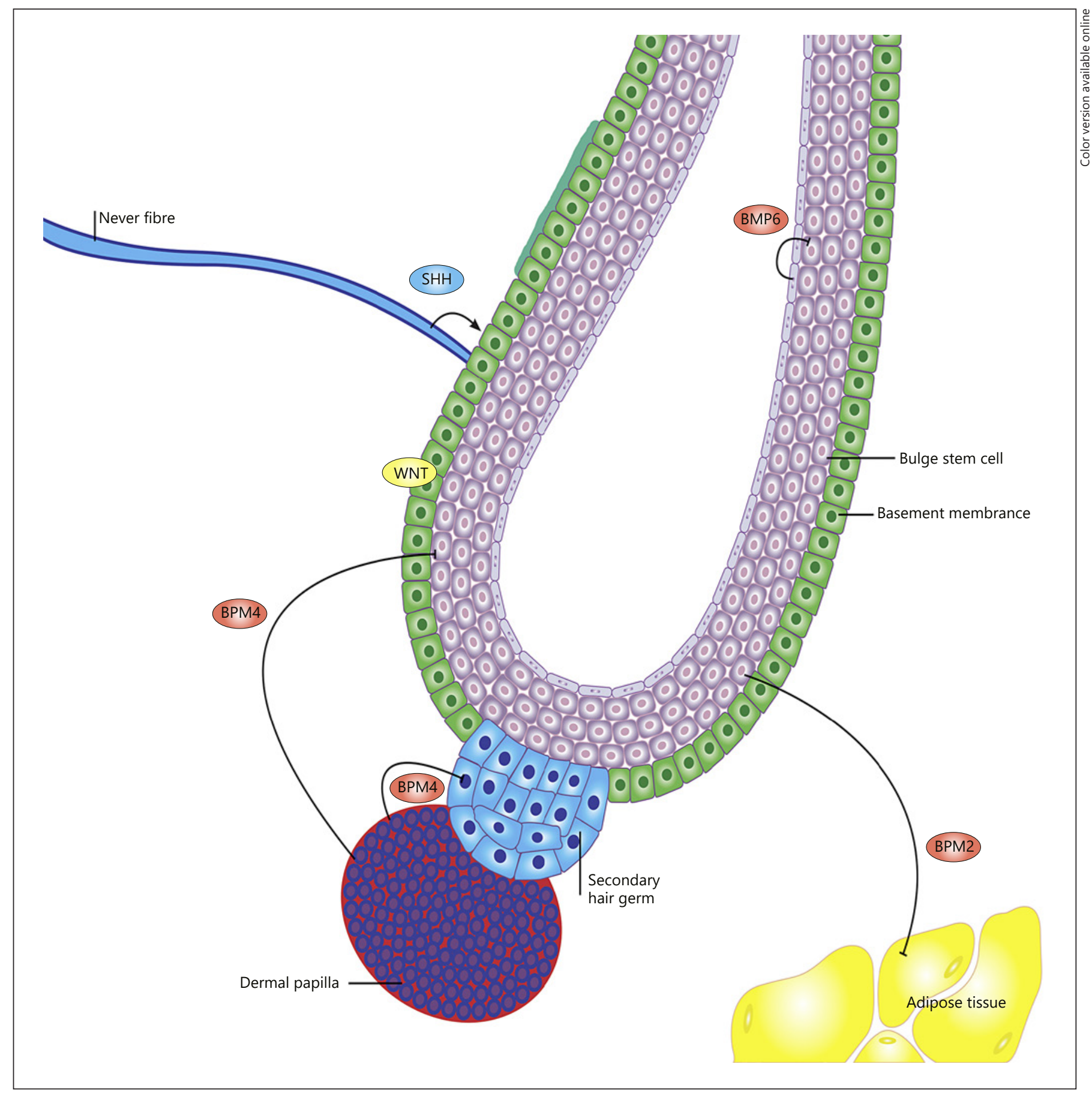

Fig. 3. The signaling pathway in the morphogenesis of hair follicles. The cell signaling pathway from major cell types, including the dermal papilla, bulge stem cells, and the adipose tissue, which control the morphogenesis and development of hair follicles. The Wnt cell signaling induces the proliferation of dermal cells and the formation of the dermal condensate. The BMP4 signaling pathway stimulates the growth of DPCs through BMP inhibitors during the anagen phase. Identifying the HFSC lineage is possible through the nerve-derived Shh signaling. BMP, bone morphogenetic protein; DPCs, dermal papilla cells; HFSC, hair follicle stem cell. 
DNA synthesis in the ORS cells and differentiates hair bulb cells into ORS cells. EGF and TGF- $\beta$ play an inhibitory role in the hair follicle formation at the initial stages of the hair follicle growth [33]. The ErbB1 receptor plays a fundamental role in regulating the development of the skin cell signaling, migration, proliferation, and differentiation.

\section{Transforming Growth Factor- $\beta$}

The TGF- $\beta$ plays a major regulatory role in the catagen phase of the hair cell cycle. Evidence suggests that the epidermal repair depends on the expression of TGF- $\beta 1$ in hair follicle cells. In mature hair follicles, TGF- $\beta 1$, TGF- $\beta 2$, and TGF- $\beta 3$ mRNAs are expressed in the IRS and ORS $[34,35]$.

\section{Hepatocyte Growth Factor}

The HGF can regulate the interaction between epithelial keratinocytes and DPCs. The HGF plays the main role in the proliferation, differentiation, and apoptosis of keratinocytes in the morphogenesis of hair follicles. Moreover, injecting the HGF into the dorsal skin of newborn mice has been proposed to induce hair follicles [36].

\section{KGF (FGF7)}

The KGF is part of the FGF family that induces the proliferation of epithelial cells. The KGF can induce the hair follicle proliferation and the differentiation of progenitor cells and promote epithelialization and wound healing, making it a key determinant of skin regeneration [37].

\section{Insulin-Like Growth Factor-1}

The IGF-1 is a multifunctional regulatory growth factor that controls cell proliferation and the survival of hair follicle cells [38]. The DPC can secrete IGF-1, IGFR-1, and IGFBPs. The results of the experimental research have shown that the exogenous IGF-1 not only changes the level of protein kinase $\mathrm{C}$ but also it is essential for maintaining hair growth.

\section{Vascular Endothelial Growth Factor}

The VEGF is a type of heparin-binding glycoprotein that stimulates the proliferation and migration of endothelial cells. During the hair growth cycle (anagen), the VEGF induces the proliferation of the capillary network [39]. The VEGF overexpression in the ORS of hair follicles accelerates the hair regrowth.

Maintaining Hair Inductivity in Human Dermal Papilla Cells

\section{Other Growth Factors}

A large body of literature shows that DPCs increase the FGF expression during hair development [40]. The exogenous FGF7 or FGF10 can also stimulate the proliferation of hair follicle keratinocytes [16].

\section{Role of the Dermal Papilla Signaling in the Hair Morphogenesis}

DPCs cannot divide by themselves; the number of neighboring cells of the dermal sheath, however, increases during the hair growth cycle [41]. Disruptions to the $\beta$-catenin signaling of DPCs inhibit the growth of hair follicles [41]. The $\beta$-catenin signaling of dermal papilla controls other signaling pathways, including FGF7 and FGF10, which regulate the growth of epithelial cells in hair follicles.

The Wnt cell signaling is the primitive dermal induction pathway for developing hair placodes. As an inhibitor of the Wnt signaling, Dkk-1 lies in the interfollicular dermis [16]. Other dermal signaling pathways with a key role in the hair morphogenesis include the FGF, the BMP, and the Noggin. The indirect function of Noggin inhibits the activity of the BMP4 and induces telogen-anagen transition [42]. In addition, the Shh signaling and the platelet-derived growth factor-A (PDGF-A) can induce the formation of dermal papilla and dermal sheath [29, 43].

\section{Isolation and Culture Methods of DPCs}

Surgical microdissection and explant culture constitute common techniques for isolating DPCs. In the surgical microdissection and explant culture, an inverted end bulb of hair follicles is placed onto a $35-\mathrm{mm}$ dish. These techniques are, however, difficult and time-consuming [44]. Enzyme digestion is a more effective method than enzymatic microdissection in the isolation of DPCs from hair follicles and their cultivation. Compared to microdissection, enzymatic isolation has shown a much higher adherence rate and growth for dermal papilla. In enzymatic isolation, human hair follicles were digested by common enzymes such as dispase and collagenase with a minor disruption $[45,46]$, and part of the hair (one-third inferior) as the dermal papilla was rinsed in collagenase I $(0.1 \%)$ at $37^{\circ} \mathrm{C}$ for $4 \mathrm{~h}$. Figure 4 shows how DPCs were cultivated in a humidified atmosphere of $5 \% \mathrm{CO}_{2}$ at $37^{\circ} \mathrm{C}$ on 6 wells using DMEM/F12, $10 \% \mathrm{FBS}, 50 \mu \mathrm{g} / \mathrm{mL}$ of pen- 


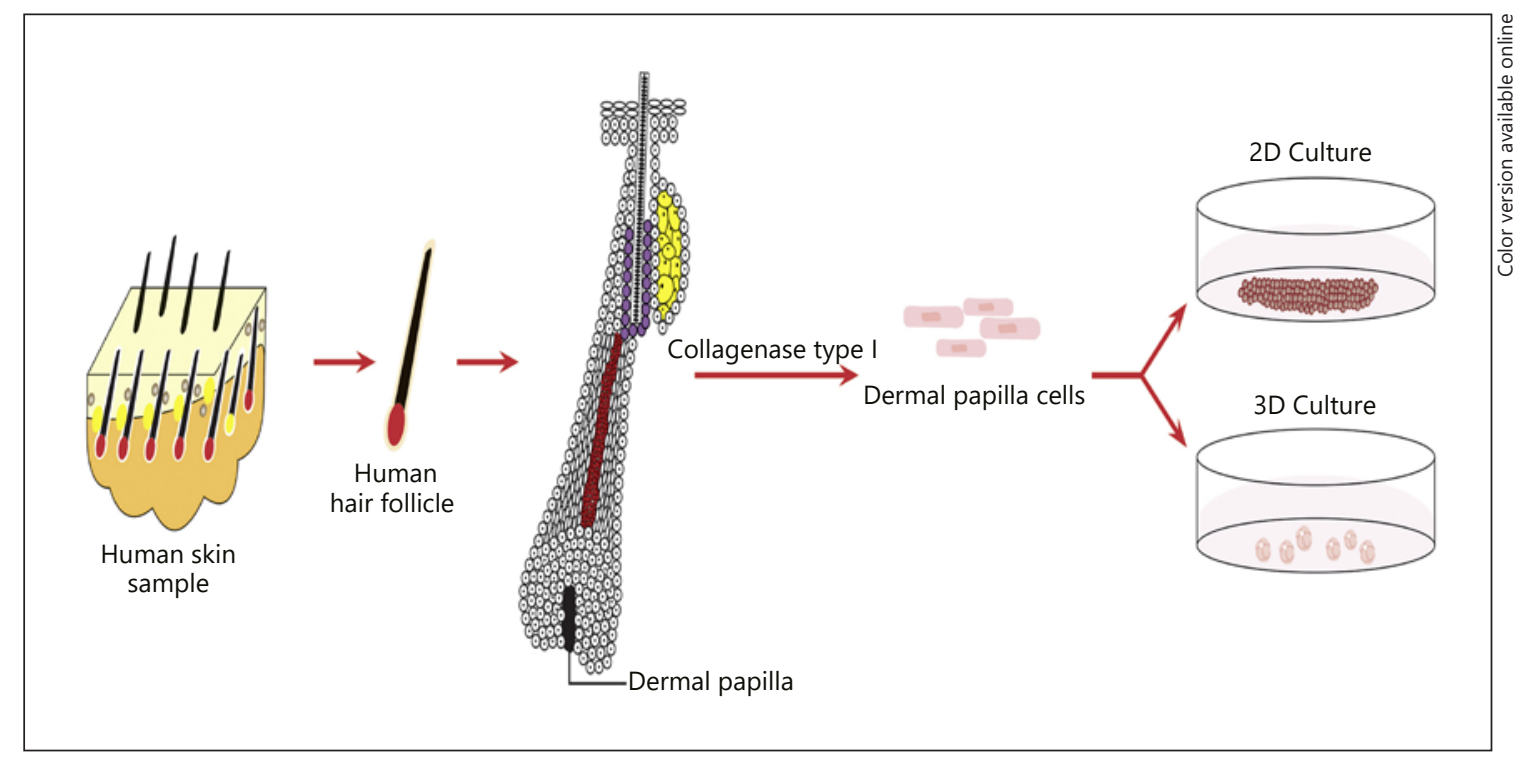

Fig. 4. A schematic view of enzymatic methods used for the isolation and cultivation of human DPCs. The human hair dermal papilla is digested by collagenase type I. DPCs, dermal papilla cells.

icillin/streptomycin, and $50 \mu \mathrm{g} / \mathrm{mL}$ of L-glutamine [46]. Cultured DPCs also showed multilayered aggregates in the first few passages. The inductivity and proliferative capacity of DPCs gradually decreased with the number of passages $[47,48]$. However, enzymatic method would disrupt specified extracellular matrix microenvironment, which is fundamental for maintaining hair inductive features. Therefore, lots of effort has been made to find out an alternative in vitro expansion procedure to preserve hair inductive ability of DPCs [49].

One approach for preserving hair inductive ability of DPCs consists in the establishment of 3D sphere cultures. The hair inductivity of dermal papilla appears to be correlated with the aggregate formation in the DPC culture. According to Figure 4, approximately 3,000 DPCs per 10 $\mu \mathrm{L}$ in the $3 \mathrm{D}$ spheroid culture were placed onto 96 lowadherent wells or inverted lids of petri dishes.

\section{Cellular and Molecular Characterization of DPCs}

As shown in Figure 5, the following marker proteins are commonly used to identify DPCs.

\section{Alkaline Phosphatase}

The hair follicle inductivity in human dermal papilla has been detected with alkaline phosphatase (ALP). The maximal level of ALP activity in the dermal papilla has been found in the early anagen. A decreased expression of ALP in the cultured DPCs after the passage has been reported as the loss of the hair inductivity in dermal papilla and dermal sheath [21].

\section{$\alpha$-Smooth Muscle Actin}

The expression of $\alpha$-smooth muscle actin ( $\alpha$-SMA) has been identified in part of the DS rather than in the dermal papilla [50]. $\alpha$-SMA is therefore an in vivo marker of the DS and an in vitro marker of both the dermal papilla and DS.

\section{Versican}

As a specific marker of the dermal papilla, versican is expressed during the anagen phase. The dermal papilla loses the versican expression in the androgenetic alopecia, as a low expression of versican has been reported in DS cells in this condition [51, 52]. The induction and maintenance of the anagen stage constitute the main role of versican in hair growth [53].

\section{CD133}

The expression of CD133 in the dermal papilla induces the hair follicle neogenesis. CD133 is a stem cell marker that is expressed in DPCs in the early anagen [54]. 
Fig. 5. A summary of different markers of the DPC population. Marker proteins commonly used to identify dermal papilla include ALP, a-SMA, versican, and CD133. DPC, dermal papilla cell; ALP, alkaline phosphatase; $\alpha$-SMA, $\alpha$-smooth muscle actin.

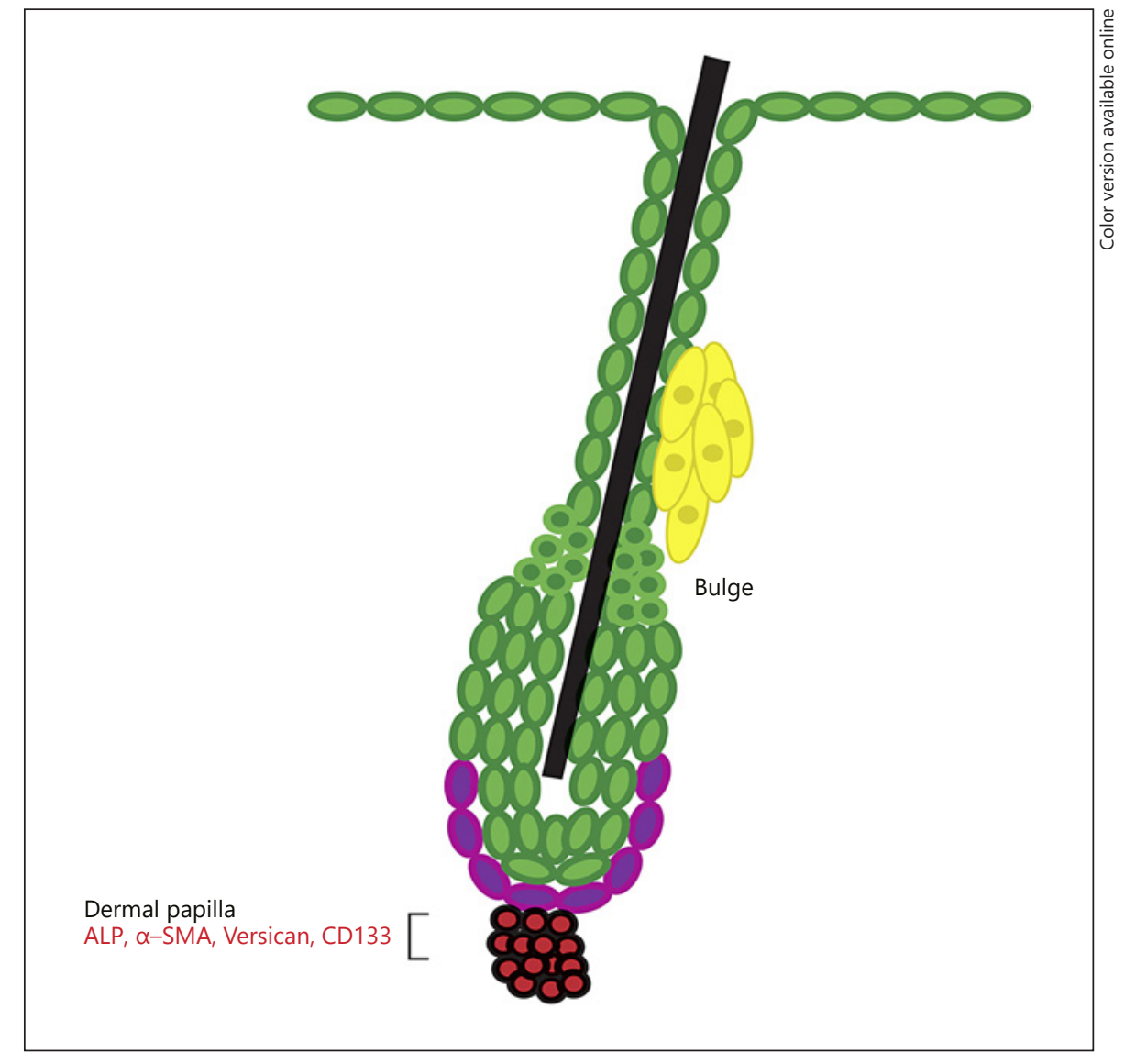

\section{Effective Methods for Maintaining the Potential Hair Inductivity in the DPC Culture}

DPCs generally cause the loss of the inductive capacity of hair follicles after a few passages $[55,56]$. Several in vitro approaches have been used to maintain the hair inductive capacity of DPCs, which are classified as follows:

\section{D Sphere Culture System}

One of the alternative in vitro approach to multiply trichogenic ability of DPCs is the establishment of 3D sphere cultures, which help to reestablish cell-cell contacts and increase in vivo DP hair inductive ability [20, 57].

The cell aggregation of dermal papilla and the activation of certain cell signaling pathways were found to enhance the inductive capacity of hair follicles. 3D culture methods, including culture systems such as HydroCell plate for sphere formation or hanging drop method, offer appropriate systems for the cultivation of DPCs, the maintenance of the inductive capacity of hair, and the ex- pression of specific markers of the dermal papilla in both humans and rodents [58]. However, there are some main obstacle in these methods such as controlling the size and cell density and the problems in the commercialization or mass production for clinical therapy.

Tissue Engineering Strategies for Human Hair Follicle Regeneration

Biodegradability, biocompatibility, and cost-effectiveness are essential features of scaffolds for hair follicle regeneration, which has been performed using different natural and synthetic scaffolds, including hydrophilic polyvinyl alcohol, chitosan and hydrogel [59-61] silk-gelatin, hyaluronic acid, and collagen [62]. In another study, in vitro human hair follicles similar to vellus were recreated with a mixture of DPCs, keratinocytes, and melanocytes in a collagen scaffold, and named "microfollicles" [49]. In a recent study, combination of hydrogel scaffold with mouse adult DPCs and epidermal cells enhances de novo hair follicle formation in mice model. 
Some of the natural and individual biomaterials used as scaffolds for the ingenious treatment of hair loss include PRP $[6,63,64]$. The components of PRP include the PDGF, the FGF, and the VEGF, which can induce proliferation, differentiation, and maintenance of the hair inductivity in DPCs [65].

\section{Conditioned Medium and Cell Signaling Approach}

Under conventional cell culture conditions, DPCs rapidly lose their inductivity for new hair formation. The limitations of conventional DPCs culture have made them commercially infeasible. Recent improvements in the cultivation of DPCs have helped maintain the hair inductivity; for instance, the co-culture of DPCs with keratinocyte-conditioned media can support both the expansion of DPCs and the maintenance of the trichogenic ability through multiple passages. Appropriate cell types obtained from the keratinocyte-conditioned medium such as keratinocytes or ADSCs can be more convenient and accessible for inducing trichogenicity in hair follicles $[66,67]$.

Conditioned media can be isolated from allogeneic and 3D cell cultures of tissues and organs. Being frozen and concentrated through ultrafiltration prior to their use constitutes a feature of conditioned media. The concentration of different cell-secreted factors can be optimized in terms of the number of cells and the duration of culture. Establishing a master cell bank can help with the manufacture of large amounts of conditioned media, which can be obtained from monolayer cultures, $3 \mathrm{D}$ cell cultures, and tissue cultures. The concentration optimization of cell-secreted factors for conditioned media depends on the culture duration, the number of cells, and the volume of the culture medium. Concentrated conditioned media made through ultrafiltration can induce and maintain the trichogenicity of hair follicles [36]. Conditioned media derived from terminally differentiated cells or committed progenitor cells from a source other than epidermal cells can also maintain the inductivity of the dermal papilla during the expansion phase of cell culture.

ADSC-conditioned media containing different types of growth factors, including KGF, VEGF, PDGF and HGF, facilitate hair growth. The advantages of using conditioned media compared to the application of cell therapy in hair regeneration include simple production, packaging, and storage. The short half-life of conditioned medium growth factors is considered a disadvantage in regenerative medicine [36].
The optimal plating density of the dermal papilla allows the maintenance of the hair inductivity with the dermal papilla in long-term cultures. Using a co-culture system and epidermal and non-epidermal feeder cells, for example, mesoderm-derived cells such as prostate epithelial cells and/or endoderm-derived cells, helps retain the hair inductive phenotype of DPCs with a cell-cell contact and hair inductive cell factors $[68,69]$. Using transwell plates in co-culture systems prevents the contamination of hair inductive cells by feeder cells.

Another technique is to use a co-culture of DPCs with an increased amount of the Wnt protein or a factor inducing the Wnt cell signaling in the culture medium $[70,71]$. During the period of losing hair inductivity of DPC, this feature can be restituted if appropriate molecular substances are provided; for example, Wnt and BMP cell signaling have been demonstrated to maintain hair follicle inductivity in cultured rodent papilla cells [15, 21]. Similar strategies such as soluble growth factors or keratinocyte-conditioned medium have been used to restore the hair inductivity of human DPCs $[18,62]$.

Using these 2 methods entails a series of problems involving the manufacturing process and costs. The co-culture of human dermal papilla with keratinocytes is costly and requires the establishment of a cell bank and accurate quality control tests for safety. The co-culture of DPCs with the exogenous Wnt protein is associated with potential problems and the stability of the Wnt gene expression during the cell culture expansion $[49,50]$.

Normal CD133+ DPCs have been found to possess the properties of inductive hair follicles. The maintenance of the trichogenicity of CD133+ DPCs has not been yet evaluated in in vitro cultures [72]. The relationship between the overexpression of $\beta$-catenin cell signaling and CD133+ dermal papilla enhances the maintenance of the trichogenic ability of cultivated dermal papilla and in vivo hair formation.

Using activated 5\% PRP enhances the hair follicle inductivity and the proliferation of dermal papilla culture in humans and mice. Higher concentrations of 5\% PRP have been found to be ineffective in the proliferation and maintenance of the dermal papilla hair inductivity. Certain secretion growth factors, including the FGF2 and PDGF, in activated PRP can play the main role in the proliferation and hair inductivity of DPCs [73, 74].

Encapsulating the dermal papilla in PRP gel scaffolds with endogenous growth factors can support the trichogenicity of DPCs in hair follicle tissue engineering. Low mechanical properties and rapid degradation constitute the main shortcomings of PRP scaffolds [34]. Cross-link- 
ing and fibrinogen content regulation improve the mechanical properties and stiffness of PRP gel scaffolds in hair tissue engineering.

Treating DPCs with herbal extracts plays a major role in activating the Akt pathway in humans. This pathway regulates the survival, proliferation, and inductivity of DPCs [75]. The result of a recent study has demonstrated that pharmacological modulation of JAK-STAT pathway was shown to improve trichogenic ability of cultured human DPCs.

Cultivation of hDPCs with glycogen synthase kinase$3 \beta$ inhibitor showed activation of $\mathrm{Wnt} / \beta$-catenin signaling and demonstrated stable hair inductive ability when transplanted with murine epidermal cell suspension [76]. Culture of DPCs with keratinocyte conditioned medium could maintain hair inductivity after 90 passages without losing their hair induction [67]. Evidence of experimental study have shown that basic FGF, same as the Wnt/ $\beta$ catenin signaling, induces hair growth $[77,78]$.

\section{Exosome Approach for Human Hair Follicle Regeneration}

Exosomes are two-layered micro-sized vesicles containing different types of protein, including fusion proteins and transport proteins, that is, annexins and flotillin; heat shock proteins, that is, HSP and HSP70; CD proteins, that is, CD9 and CD81; and mRNA and microRNA with a diameter of $40-100 \mathrm{~nm}$, which can communicate with another cell and induce cellular function in a new location. Exosomes were isolated from serum-free conditioned media using different methods such as differential ultracentrifuge, chromatography, filtration, immunological separation, and polymer-based precipitation.

A defective dermal papilla signaling causes hair loss in the androgenetic alopecia. Mesenchymal stem cell derived extracellular vesicles (MSC-EVs) activate the hair inductivity of DPCs and stimulate Akt phosphorylation and increase Bcl-2 in dermal papilla, which regulates the DPC proliferation [79].

The in vivo results obtained from the MSC-EVs treatment of hair loss in mice showed the conversion of the telogen phase of hair follicles to the anagen phase and the induction of hair follicles with dermal papilla. Also MSC$\mathrm{EV}$ approach promotes the growth of hair follicles with secretion of VEGF, HGF, and IGF-1 [80]. The report has shown that the injection of MSC-EVs into C57BL/6 mice induced the conversion from telogen phase of hair follicles to anagen [79]. However, using these appropriate rodent experiments into the human model has been a ratelimiting step with these approaches.

Maintaining Hair Inductivity in Human Dermal Papilla Cells

\section{Conclusion}

Hair loss is globally a medical problem from which many women and men in the world suffer. DPCs play a key role in regulating hair growth and hair regeneration. Different issues involved in the hair neogenesis include the dermal papilla isolation and cultivation, the maintenance of the hair inductivity of the dermal papilla, the provision of appropriate niche, exogenous growth factors, and cell signals. Numerous studies have therefore focused on effective methods in culturing human dermal papilla and maintaining the induction properties of hair follicles.

The results of experimental studies have shown that DPCs rapidly lose their inductivity for new hair formation in conventional 2D cell cultures [9]. In the surgical microdissection and explant culture methods, an inverted end bulb of hair follicles is placed onto a cell culture dish. In enzymatic isolation, the human hair follicle is digested by common enzymes such as dispase and collagenase with a minor disruption. Research suggests that the hair inductive capacity of the dermal papilla is significantly reduced by the $2 \mathrm{D}$ in vitro expansion. The results obtained from the $2 \mathrm{D}$ culture of vibrissal DPCs in rats showed loss of the hair inductive activity in later passages [81, 82]. Losing the hair inductive properties after over 10 passages is therefore considered a major problem of the 2D culture of DPCs. The disorders of two-dimensional DPC cultures to take the appropriate characteristics of the in-vivo microenvironment, cell communication, cell signaling, and metabolic functions extremely affect the hair follicle inductive ability of DPCs [26].

The hanging drop and sphere culture of the dermal papilla not only morphologically simulates the dermal papilla but also provides a similar molecular level of the normal intact hair dermal papilla. In hanging drop culture, DPCs in the Matrigel or alginate drop are placed onto the low-adherent inverted lid of a petri dish. Compared to the conventional $2 \mathrm{D}$ culture, the loss of the a-SMA expression in the spheroid culture of the dermal papilla showed reductions in the proliferative capacity, although the re-differentiating properties to papilla-like phenotypes were maintained. The expansion of the dermal papilla in a $3 \mathrm{D}$ microenvironment was found to restore the expression of versican through the partial recovery of the inductive capacity of DPCs. The 3D culture of DPCs in the alginate sphere was found to increase the main development cell signaling of hair follicles (SOX2, ALPL, Noggin, BMP4, and versican).

Skin Pharmacol Physiol 2020;33:280-292

DOI: $10.1159 / 000510152$ 
3D cultures appear to be useful for maintaining the potential in vitro and in vivo hair activity of the dermal papilla; nevertheless, reducing the rate of growth and proliferation of DPCs constitutes a limitation of this method. Controlling the cell density and size in clinical applications also constitute the problems of using low-adherent plates and hanging drop techniques for the 3D culture of DPCs.

The number of growth factors, including HGF, IGF, VEGF, PDGF, FGF, and KGF, and receptors affecting the development of hair follicles or the dermal papilla have been found to be different. The functional mechanism of the growth factors depends on the phosphorylation of tyrosine.

Stem cell-conditioned media containing different types of cytokine and growth factors such as the HGF, the PDGF, the KGF, and the VEGF play a critical role in hair growth and maintenance of hair inductive properties of dermal papilla. A growth factor cocktail of keratinocyteconditioned media such as VEGF and IGF-1 was found to significantly increase the proliferation of DPCs and induce hair follicles in mouse models [13]. The results of experimental studies showed that Wntla- and BM-MSCconditioned media stimulate the conversion of hair follicles from the telogen to anagen phase. This cocktail was also found to significantly increase the number of hair follicles in mice [45]. The short half-life of cytokines and growth factors in stem cell-conditioned media is a disadvantage of this method from a clinical application perspective [83].

Cell-cell interactions play a key role in different physiological processes. Recently conducted research has attributed the efficacy of cell therapy to the paracrine function. Extracellular vesicles and exosomes can therefore play the main role in modulating hair growth. The protocol of the exosome isolation of conditioned media comprises a serial low-speed centrifugation and ultracentrifugation. A conditioned medium was centrifuged at $300 \mathrm{~g}$ for $10 \mathrm{~min}$ to remove the cells. The dead cells were removed by centrifuging at $2,000 \mathrm{~g}$ for $10 \mathrm{~min}$. The supernatant was transferred to a $15-\mathrm{mL}$ conical tube and then centrifuged at 10,000 $g$ to remove the cell debris. In the next step, the supernatant underwent ultracentrifugation at $100,000 \mathrm{~g}$ for $2 \mathrm{~h}$ to obtain exosome containing proteins. The supernatant was again collected in a new tube and centrifuged at $100,000 \mathrm{~g}$ for $2 \mathrm{~h}$ at $4^{\circ} \mathrm{C}$. The pellet now including the exosomes was diluted in $\times 1$ PBS and stored at $-80^{\circ} \mathrm{C}$ for future use.

Investigating the effects of MSC-EVs on hair regrowth in C57BL/6 mice demonstrated the induction of the anagen phase similar to standard methods such as minoxidil therapy. Activating the Akt pathway and increasing the level of Bcl-2 in DPCs with MSC-EVs can extend the durability of DPCs. Research suggests the positive role of MSC-EVs in the secretion of VEGF and IGF-1 of DPCs and the induction of hair follicle growth. Increasing the gene expression of VEGF and IGF-1 induces hair growth and the hair inductive capacity of the dermal papilla. The exosomes derived from human hair dermal papilla activate the $\mathrm{Wnt} / \beta$-catenin and Shh cell signaling pathways to regulate hair follicle growth. The results of the EV treatment of hair loss in mice appeared promising for improving the hair induction capacity of DPCs. Our result has shown HHORSC-Exo and ASC-Exo as new methods to support hair inductivity of DPCs and improve the outcome for the treatment of hair loss.

Compared to other approaches used for maintaining the in vitro and in vivo hair inductivity of DPCs, exosome-based treatment is recommended as an efficient method. The advantages of using exosomes in experimental and clinical applications include inducing endogenous mechanisms, simple processing, long-term storage, and reduced risks of immune reactions.

Using a different strategy for maintaining the hair inductive capacity of the dermal papilla can provide an appropriate discovering method for therapeutic objectives of hair regeneration. Further studies are recommended that be conducted using a different strategy with different compounds and adjuvants to broaden the existing knowledge on maintaining the hair inductivity of the dermal papilla.

\section{Acknowledgements}

The authors would like to thank the Skin and Stem Cell Research Center and Royan Institute for financially supporting this project. This research was the thesis of a $\mathrm{PhD}$ student from Tehran University of Medical Science.

\section{Conflicts of Interest Statement}

The authors declared no conflicts of interest regarding the publication of the present article.

\section{Author Contributions}

E.T. proposed the idea of the article and performed a review of literature. N.A. and M.A.N. drafted and/or critically revised the paper. All the authors edited and approved the final revision of this paper for submission, and participated in the finalization of the manuscript and approval of the final draft. 


\section{References}

1 Gomolin A, Litvinov IV, Netchiporouk E. Oral minoxidil: a possible new therapy for androgenetic alopecia. J Cutan Med Surg. 2020; 24(1):88-9.

2 Tai T, Kochhar A. Physiology and medical treatments for alopecia. Facial Plast Surg Clin North Am. 2020;28(2):149-59.

3 Rho SS, Park SJ, Hwang SL, Lee MH, Kim CD, Lee IH, et al. The hair growth promoting effect of Asiasari radix extract and its molecular regulation. J Dermatol Sci. 2005;38(2):89-97.

4 Tong T, Kim N, Park T. Topical application of oleuropein induces anagen hair growth in telogen mouse skin. PLoS One. 2015;10(6): e0129578.

5 Patel S, Sharma V, Chauhan NS, Thakur M, Dixit VK. Hair growth: focus on herbal therapeutic agent. Curr Drug Discov Technol. 2015;12(1):21-42.

6 Khatu SS, More YE, Gokhale NR, Chavhan DC, Bendsure N. Platelet-rich plasma in androgenic alopecia: myth or an effective tool. J Cutan Aesthet Surg. 2014;7(2):107-10.

7 Li ZJ, Choi HI, Choi DK, Sohn KC, Im M, Seo YJ, et al. Autologous platelet-rich plasma: a potential therapeutic tool for promoting hair growth. Dermatol Surg. 2012;38(7 Pt 1): 1040-6.

8 Godse K. Platelet rich plasma in androgenic alopecia: where do we stand? J Cutan Aesthet Surg. 2014;7(2):110-1.

9 Alves R, Grimalt R. Randomized placebocontrolled, double-blind, half-head study to assess the efficacy of platelet-rich plasma on the treatment of androgenetic alopecia. Dermatol Surg. 2016;42(4):491-7.

10 Stefanis AJ, Groh T, Arenbergerova M, Arenberger P, Bauer PO. Stromal vascular fraction and its role in the management of alopecia: a review. J Clin Aesthet Dermatol. 2019;12(11): 35-44.

11 Gentile P, Garcovich S. Advances in regenerative stem cell therapy in androgenic alopecia and hair loss: Wnt pathway, growth-factor, and mesenchymal stem cell signaling impact analysis on cell growth and hair follicle development. Cells. 2019;8(5):466.

12 Epstein GK, Epstein JS. Mesenchymal stem cells and stromal vascular fraction for hair loss: current status. Facial Plast Surg Clin North Am. 2018;26(4):503-11.

13 Won CH, Jeong YM, Kang S, Koo TS, Park $\mathrm{SH}$, Park KY, et al. Hair-growth-promoting effect of conditioned medium of high integrin a6 and low CD 71 (a6bri/CD71dim) positive keratinocyte cells. Int J Mol Sci. 2015;16(3) 4379-91.

14 Sharma R, Ranjan A. Follicular unit extraction (FUE) hair transplant: curves ahead. J Maxillofac Oral Surg. 2019;18(4):509-17.

15 Rishikaysh P, Dev K, Diaz D, Qureshi WM, Filip S, Mokry J. Signaling involved in hair follicle morphogenesis and development. Int J Mol Sci. 2014;15(1):1647-70.
16 Greco V, Chen T, Rendl M, Schober M, Pasolli HA, Stokes N, et al. A two-step mechanism for stem cell activation during hair regeneration. Cell Stem Cell. 2009;4(2):155-69.

17 Reynolds AJ, Jahoda CA. Hair matrix germinative epidermal cells confer follicle-inducing capabilities on dermal sheath and high passage papilla cells. Development. 1996;122(10): 3085-94.

18 Qiao J, Zawadzka A, Philips E, Turetsky A, Batchelor S, Peacock J, et al. Hair follicle neogenesis induced by cultured human scalp dermal papilla cells. Regen Med. 2009;4(5):667-76.

19 Birgersdotter A, Sandberg R, Ernberg I. Gene expression perturbation in vitro: a growing case for three-dimensional (3D) culture systems. Semin Cancer Biol. 2005;15(5):405-12.

20 Kang BM, Kwack MH, Kim MK, Kim JC, Sung YK. Sphere formation increases the ability of cultured human dermal papilla cells to induce hair follicles from mouse epidermal cells in a reconstitution assay. J Invest Dermatol. 2012;132(1):237-9.

21 Rendl M, Polak L, Fuchs E. BMP signaling in dermal papilla cells is required for their hair follicle-inductive properties. Genes Dev. 2008;22(4):543-57.

22 Wu P, Zhang Y, Xing Y, Xu W, Guo H, Deng F, et al. The balance of Bmp6 and Wnt10b regulates the telogen-anagen transition of hair follicles. Cell Commun Signal. 2019;17(1):16.

23 Galbraith H. Fundamental hair follicle biology and fine fibre production in animals. Animal. 2010;4(9):1490-509.

24 Yu BD, Mukhopadhyay A, Wong C. Skin and hair: models for exploring organ regeneration. Hum Mol Genet. 2008;17(R1):R54-9.

25 Buffoli B, Rinaldi F, Labanca M, Sorbellini E, Trink A, Guanziroli E, et al. The human hair: from anatomy to physiology. Int J Dermatol. 2014;53(3):331-41

26 Alonso LC, Rosenfield RL. Molecular genetic and endocrine mechanisms of hair growth. Horm Res. 2003;60(1):1-13.

27 Driskell RR, Clavel C, Rendl M, Watt FM Hair follicle dermal papilla cells at a glance. J Cell Sci. 2011;124(Pt 8):1179-82.

28 Shimomura Y, Christiano AM. Biology and genetics of hair. Annu Rev Genomics Hum Genet. 2010;11.109-32.

29 Woo WM, Zhen HH, Oro AE. Shh maintains dermal papilla identity and hair morphogenesis via a Noggin-Shh regulatory loop. Genes Dev. 2012;26(11):1235-46.

30 Ohyama M, Kobayashi T, Sasaki T, Shimizu A, Amagai M. Restoration of the intrinsic properties of human dermal papilla in vitro. J Cell Sci. 2012;125(Pt 17):4114-25

31 Peus D, Pittelkow MR. Growth factors in hair organ development and the hair growth cycle. Dermatol Clin. 1996;14(4):559-72.

32 Choi N, Choi J, Kim JH, Jang Y, Yeo JH, Kang J, et al. Generation of trichogenic adipose-derived stem cells by expression of three factors. J Dermatol Sci. 2018;92(1):18-29.
33 Alexandrescu DT, Kauffman CL, Dasanu CA. Persistent hair growth during treatment with the EGFR inhibitor erlotinib. Dermatol Online J. 2009;15(3):4.

34 Sugawara K, Kizaki K, Herath CB, Hasegawa $\mathrm{Y}$, Hashizume K. Transforming growth factor beta family expression at the bovine feto-maternal interface. Reprod Biol Endocrinol. 2010;8.120.

35 Inui S, Itami S. Androgen receptor transactivity is potentiated by TGF- $\beta 1$ through Smad3 but checked by its coactivator Hic-5/ARA55 in balding dermal papilla cells. J Dermatol Sci. 2011;64(2):149-51

36 Fushimi T, Inui S, Ogasawara M, Nakajima T, Hosokawa K, Itami S. Narrow-band red LED light promotes mouse hair growth through paracrine growth factors from dermal papilla. J Dermatol Sci. 2011;64(3):246-8.

37 Radek KA, Taylor KR, Gallo RL. FGF-10 and specific structural elements of dermatan sulfate size and sulfation promote maximal keratinocyte migration and cellular proliferation. Wound Repair Regen. 2009;17(1):118-26.

38 Forbes BE, McCarthy P, Norton RS. Insulinlike growth factor binding proteins: a structural perspective. Front Endocrinol. 2012;3: 38.

39 Lee GS, Hong EJ, Gwak KS, Park MJ, Choi KC, Choi IG, et al. The essential oils of Chamaecyparis obtusa promote hair growth through the induction of vascular endothelial growth factor gene. Fitoterapia. 2010;81(1): $17-24$.

40 Enshell-Seijffers D, Lindon C, Wu E, Taketo MM, Morgan BA. Beta-catenin activity in the dermal papilla of the hair follicle regulates pigment-type switching. Proc Natl Acad Sci U S A. 2010;107(50):21564-9.

41 Enshell-Seijffers D, Lindon C, Kashiwagi M, Morgan BA. $\beta$-Catenin activity in the dermal papilla regulates morphogenesis and regeneration of hair. Dev Cell. 2010;18(4):633-42.

42 Plikus MV, Mayer JA, de la Cruz D, Baker RE, Maini PK, Maxson R, et al. Cyclic dermal BMP signalling regulates stem cell activation during hair regeneration. Nature. 2008; 451(7176):340-4

43 Karlsson L, Bondjers C, Betsholtz C. Roles for PDGF-A and sonic hedgehog in development of mesenchymal components of the hair follicle. Development. 1999;126(12):2611-21.

44 Horne KA, Jahoda CA. Restoration of hair growth by surgical implantation of follicular dermal sheath. Development. 1992;116(3): 563-71.

45 Wu JJ, Liu RQ, Lu YG, Zhu TY, Cheng B, Men $\mathrm{X}$. Enzyme digestion to isolate and culture human scalp dermal papilla cells: a more efficient method. Arch Dermatol Res. 2005; 297(2):60-7. 
46 Nilforoushzadeh M, Rahimi Jameh E, Jaffary F, Abolhasani E, Keshtmand G, Zarkob H, et al. Hair follicle generation by injections of adult human follicular epithelial and dermal papilla cells into nude mice. Cell J. 2017;19(2): 259-68.

47 Topouzi H, Logan NJ, Williams G, Higgins CA. Methods for the isolation and 3D culture of dermal papilla cells from human hair follicles. Exp Dermatol. 2017;26(6):491-6.

48 Higgins CA, Chen JC, Cerise JE, Jahoda CA, Christiano AM. Microenvironmental reprogramming by three-dimensional culture enables dermal papilla cells to induce de novo human hair-follicle growth. Proc Natl Acad Sci U S A. 2013;110(49):19679-88.

49 Castro AR, Logarinho E. Tissue engineering strategies for human hair follicle regeneration: how far from a hairy goal? Stem Cells Transl Med. 2020;9(3):342-50.

50 Jahoda CA, Reynolds AJ, Chaponnier C, Forester JC, Gabbiani G. Smooth muscle alphaactin is a marker for hair follicle dermis in vivo and in vitro. J Cell Sci. 1991;99(Pt 3): 627-36.

51 Soma T, Tajima M, Kishimoto J. Hair cyclespecific expression of versican in human hair follicles. J Dermatol Sci. 2005;39(3):147-54.

52 Yang Y, Li Y, Wang Y, Wu J, Yang G, Yang T, et al. Versican gene: regulation by the $\beta$-catenin signaling pathway plays a significant role in dermal papilla cell aggregative growth. J Dermatol Sci. 2012;68(3):157-63.

53 Kim SR, Cha SY, Kim MK, Kim JC, Sung YK. Induction of versican by ascorbic acid 2-phosphate in dermal papilla cells. J Dermatol Sci. 2006;43(1):60-2.

54 Ito Y, Hamazaki TS, Ohnuma K, Tamaki K, Asashima M, Okochi H. Isolation of murine hair-inducing cells using the cell surface marker prominin-1/CD133. J Invest Dermatol. 2007;127(5):1052-60.

55 Aoi N, Inoue K, Chikanishi T, Fujiki R, Yamamoto $\mathrm{H}$, Kato H, etal. 1 $\alpha, 25$-dihydroxyvitamin D3 modulates the hair-inductive capacity of dermal papilla cells: therapeutic potential for hair regeneration. Stem Cells Transl Med. 2012;1(8):615-26.

56 Xiao SE, Miao Y, Wang J, Jiang W, Fan ZX, Liu XM, et al. As a carrier-transporter for hair follicle reconstitution, platelet-rich plasma promotes proliferation and induction of mouse dermal papilla cells. Sci Rep. 2017;7(1): 1125.

57 Mohammadi P, Youssef KK, Abbasalizadeh S, Baharvand H, Aghdami N. Human hair reconstruction: close, but yet so far. Stem Cells Dev. 2016;25(23):1767-79.

58 Nilforoushzadeh MA, Zare M, Zarrintaj P, Alizadeh E, Taghiabadi E, Heidari-Kharaji M, et al. Engineering the niche for hair regeneration: a critical review. Nanomedicine. 2019; 15(1):70-85.

59 Higgins CA, Richardson GD, Ferdinando D, Westgate GE, Jahoda CA. Modelling the hair follicle dermal papilla using spheroid cell cultures. Exp Dermatol. 2010;19(6):546-8.
60 Driskell RR, Juneja VR, Connelly JT, Kretzschmar K, Tan DW, Watt FM. Clonal growth of dermal papilla cells in hydrogels reveals intrinsic differences between Sox2-positive and -negative cells in vitro and in vivo. J Invest Dermatol. 2012;132(4):1084-93.

61 Young TH, Lee CY, Chiu HC, Hsu CJ, Lin SJ. Self-assembly of dermal papilla cells into inductive spheroidal microtissues on poly(ethylene-co-vinyl alcohol) membranes for hair follicle regeneration. Biomaterials. 2008;29(26):3521-30.

62 Kalabusheva E, Terskikh V, Vorotelyak E. Hair germ model in vitro via human postnatal keratinocyte-dermal papilla interactions: impact of hyaluronic acid. Stem Cells Int. 2017; 2017:9271869.

63 Faghihi G, Poostiyan N, Asilian A, AbtahiNaeini B, Shahbazi M, Iraji F, et al. Efficacy of fractionated microneedle radiofrequency with and without adding subcision for the treatment of atrophic facial acne scars: A randomized split-face clinical study. J Cosmet Dermatol. 2017;16(2):223-9.

64 Faghihi G, Mozafarpoor S, Asilian A, Mokhtari F, Esfahani AA, Bafandeh B, et al. The effectiveness of adding low-level light therapy to minoxidil $5 \%$ solution in the treatment of patients with androgenetic alopecia. Indian J Dermatol Venereol Leprol. 2018;84(5):54753.

65 Cervantes J, Perper M, Wong LL, Eber AE, Villasante Fricke AC, Wikramanayake TC, et al. Effectiveness of platelet-rich plasma for androgenetic alopecia: a review of the literature. Skin Appendage Disord. 2018;4(1):1-11.

66 Dong L, Hao H, Xia L, Liu J, Ti D, Tong C, et al. Treatment of MSCs with Wntla-conditioned medium activates DP cells and promotes hair follicle regrowth. Sci Rep. 2014; 4.5432 .

67 Inamatsu M, Matsuzaki T, Iwanari H, Yoshizato K. Establishment of rat dermal papilla cell lines that sustain the potency to induce hair follicles from afollicular skin. J Invest Dermatol. 1998;111(5):767-75.

68 Driskell RR, Giangreco A, Jensen KB, Mulder KW, Watt FM. Sox2-positive dermal papilla cells specify hair follicle type in mammalian epidermis. Development. 2009; 136(16): 2815-23.

69 Veraitch O, Mabuchi Y, Matsuzaki Y, Sasaki $\mathrm{T}$, Okuno $\mathrm{H}$, Tsukashima A, et al. Induction of hair follicle dermal papilla cell properties in human induced pluripotent stem cell-derived multipotent LNGFR $(+)$ THY-1(+) mesenchymal cells. Sci Rep. 2017;7.42777.

70 Chalisserry EP, Nam SY, Park SH, Anil S. Therapeutic potential of dental stem cells. J Tissue Eng. 2017;8:2041731417702531.
71 Kishimoto J, Burgeson RE, Morgan BA. Wnt signaling maintains the hair-inducing activity of the dermal papilla. Genes Dev. 2000;14(10): 1181-5.

72 Zhou L, Xu M, Yang Y, Yang K, Wickett RR, Andl T, et al. Activation of $\beta$-catenin signaling in CD133-positive dermal papilla cells drives postnatal hair growth. PLoS One. 2016;11(7): e0160425.

73 Miao Y, Feng CB, Zhang ZD, Li ZH, Xiao SE, Jiang JD, et al. [Effect of PRP on the proliferation of dermal papilla cells and hair follicle regeneration in mice]. Zhonghua Zheng Xing Wai Ke Za Zhi. 2013;29(2):131-5.

74 Miao Y, Sun YB, Sun XJ, Du BJ, Jiang JD, Hu ZQ. Promotional effect of platelet-rich plasma on hair follicle reconstitution in vivo. Dermatol Surg. 2013;39(12):1868-76.

75 Rastegar H, Ahmadi Ashtiani H, Aghaei M, Ehsani A, Barikbin B. Combination of herbal extracts and platelet-rich plasma induced dermal papilla cell proliferation: involvement of ERK and Akt pathways. J Cosmet Dermatol. 2013;12(2):116-22.

76 Soma T, Fujiwara S, Shirakata Y, Hashimoto $\mathrm{K}$, Kishimoto J. Hair-inducing ability of human dermal papilla cells cultured under Wnt/ $\beta$-catenin signalling activation. Exp Dermatol. 2012;21(4):307-9.

77 Osada A, Iwabuchi T, Kishimoto J, Hamazaki TS, Okochi H. Long-term culture of mouse vibrissal dermal papilla cells and de novo hair follicle induction. Tissue Eng. 2007;13(5): 975-82.

78 Zhang P, Kling RE, Ravuri SK, Kokai LE, Rubin JP, Chai JK, et al. A review of adipocyte lineage cells and dermal papilla cells in hair follicle regeneration. J Tissue Eng. 2014; 5.2041731414556850 .

79 Rajendran RL, Gangadaran P, Bak SS, Oh JM, Kalimuthu S, Lee HW, et al. Extracellular vesicles derived from MSCs activates dermal papilla cell in vitro and promotes hair follicle conversion from telogen to anagen in mice. Sci Rep. 2017;7(1):15560.

80 Kwack MH, Seo CH, Gangadaran P, Ahn BC, Kim MK, Kim JC, et al. Exosomes derived from human dermal papilla cells promote hair growth in cultured human hair follicles and augment the hair-inductive capacity of cultured dermal papilla spheres. Exp Dermatol. 2019;28(7):854-7.

81 Horne KA, Jahoda CA, Oliver RF. Whisker growth induced by implantation of cultured vibrissa dermal papilla cells in the adult rat. J Embryol Exp Morphol. 1986;97.111-24.

82 Lichti U, Weinberg WC, Goodman L, Ledbetter S, Dooley T, Morgan D, et al. In vivo regulation of murine hair growth: insights from grafting defined cell populations onto nude mice. J Invest Dermatol. 1993;101(1 Suppl): 124S-9S.

83 Pawitan JA. Prospect of stem cell conditioned medium in regenerative medicine. Biomed Res Int. 2014;2014:965849. 